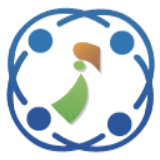

\title{
Robust Image Restoration using Non-Local Graph Based Transform and Wave Atoms
}

\author{
Arvind Kumar Ganesh ${ }^{1 *} \quad$ Ashok Kusagur ${ }^{2}$ \\ ${ }^{1}$ Telecommunication Engineering, Ramaiah Institute of Technology, India \\ ${ }^{2}$ Electrical \& Electronics Engineering, University B.D.T. College of Engineering, India \\ * Corresponding author's Email: arvind@msrit.edu
}

\begin{abstract}
The image restoration is the process of recovering the uncorrupted image from the noisy or blurred image. The aim of this method is to propose a hybrid method for effective image restoration process. The Hybrid involves in Non-Local Graph based Transform (NLGBT) learns basic vectors from the image and Wave atom technique is applied for harmonic computational analysis. In order to test the performance of the proposed system, inbuilt MATLAB image is considered and these images has been degraded with Gaussian blur, then added to the Gaussian noise. The degraded images are used to plot NLGBT and the basic vectors learn from this graph. Wave atoms coefficient has been set with the threshold values, which smoothen the image with provided vector. Instead of smoothening of the images, smoothening on the given basic vectors of the images from the NLGBT technique helps in achieving higher efficiency. The existing methods involves in smoothing of images for denoising and proposed hybrid method involves in smoothening of basic vectors from NLGBT. The result provided by the wave atom technique achieves the PSNR value of $18.57 \mathrm{~dB}$, state-of-the-art method achieves $20.08 \mathrm{~dB}$ and proposed technique achieve up to 29.02. The experimental result shows that the NLGBT and wave atoms attains high quality of the image in the restoration process compared to other existing method.
\end{abstract}

Keywords: Gaussian blur, Non-local graph based transform, PSNR, restoration, Wave atoms.

\section{Introduction}

The image restoration is one of the important topic in the image processing and this is highly studied, in which the main process is to retrieve the detailed images from noisy image [1]. The clinical process highly depends on the visual concept of the images. So, the image enhancement and denoising technique are used to increase the quality of the image [2]. The space variant image restoration or deblurring technique recover the image from the blur region and locally consistent assumption was applied to the image to generate the blur kernel for each pixel. Then the refining technique is applied to estimate the pixel using kernel function [3]. The technology development in imaging sensors, video system, digital TV and imaging sensor increases the need for efficient deblurring technique [4]. In the process of the image acquisition and transmission, the images are highly degraded due to various natural factors, which are all annoying and troublesome. Compressed sensing techniques are having its own benefits in the restoration and these requires further image processing technique to improve the image quality [5]. Linear smoothing techniques provide good results, but reduce the visual details of image, that causes the image not exploitable for further image restoration [6].

Motion blur is another issue presents in the image restoration process and this happens because of the motion of objects and cameras during exposure time. There are some areas like traffic control, medical image, astronautics and aerospace, highly depends on the details of the image and quality is affected by the image deterioration [7]. The main reasons of the image degradations are speckle noise, backscattering, relative motions and other adverse factors causes the information loss in the image [8]. Most of the methods of image 
restoration achieved high performances based on the feature selection [9] and the conventional method finds the proper channel by measuring the noise to filter out noise [10].

The feature selection plays an important role in the denoising and deblurring. The deep learning method Convolution Neural Network (CNN) help select the features Synthetic Aperture Radar (SAR) image for ship detection [11]. Akbarizadeh [12] presented the wavelet function as the feature selection and followed by Support Vector Machine (SVM) as classifier. The Local Spectral Histogram (LSH) used for the textural based segmentation and this is used to initiate the level set [13]. For feature dimensionality reduction, Random Space (RS) and Linear Discriminant Analysis and Sparse Regularisation (LDASR) is presented [14]. Another combination of feature selection method such as Cellular Automata (CA) and Fuzzy rules provides the considerable performance [15]. Nine shaped based features selection methods studied in the research [16] and the study identified that Zernike moment descriptor has better performance. The blob features were obtained using the combination of Gaussian-guided filter (GGF) with a scale invariant feature transform [17]. The fuzzy inference system was used to learn the feature and to decrease the system error in the automated lung cancer detection [18]. In this paper, NLGBT and Wave atoms techniques are used to restore the degraded image. NLGBT learns the basic vectors from the plotted graph and these vectors are used by the wave atoms to restore the image. The threshold coefficient value is set into wave atoms to classify the images for reconstruction. The wave atom smoothing the basic vectors provided by the NLGBT. These values are used to reconstruct the image with reduced noise. The inbuilt MATLAB images are used for the evaluation of the proposed method. The existing method involves in smoothing of images that requires more computational time and quality is not much preserved. So, the proposed hybrid method involves in smoothing of basic vectors of the degraded image. The outcome of the hybrid method discussed in the experimental result with comparative models.

\section{Literature review}

In this section, latest research paper related to image restoration reviewed for their contribution on the image restoration technique. The research related to the Gaussian blur degraded image restoration techniques reviewed in this section.
T. Liu, Z. Chen, S. Liu, Z. Zhang and J. Shu [19] investigated the bind deconvolution problem, which is created from the usage of true sparsity Passive millimeter wave image (PMMW) and high resolution PMMW images. This method has three main processes, (1) the important information from the images are assigned in data selection matrix, (2) sparse priori regularization is the new smoothness constraint fitted to the high-resolution image. This helps in preserving the details of the image and also suppress the noise, (3) at last, effective optimization algorithm is used for advance schemes. The details of the images obtained but the Mean Square Error is high for some images and the restored images have less accurate information.

A. Kumar, M. F. Hassan, and P. Raveendran [20] proposed a method that measure the Point Spread Function (PSF) from the degraded image. They used the extreme training method to train and geometric moments is used as feature vectors. After PSF calculation, a mathematical framework used to attain geometric modes of the restored image. A fast inverse reconstruction technique was used to convert the restored image into spatial domain. This helped to solve the problem of non-orthogonality of geometric moments and made the reconstruction process. The mathematical technique achieved higher performance compared to existing method in terms of image restoration. The proposed method failed to achieve higher SSMI value for some images in the LIVE database.

S. Suresh, D. Das, S. Lal and D. Gupta [21] proposed an effective quality enhancement of images, which is focused on the enhancement of contrast in satellite remote sensing images. This technique evaluated using many remote sensing images and it achieved highest performance compared to the existing methods. The comparison of qualitative evaluation validated in terms of Michelson contrast, which is the measure of enhancement and Discrete entropy. The pre-processing is not applied in this method and it takes more execution time than the existing methods.

Xinwu Liu, [22] introduced the method of alternating augmented Lagrangian method that contains three auxiliary variables for solving the objective function of TGV-KL model. The other advantage of this method is the optimization method includes TGV norm, that recovers the sharpness of image and avoid stair casting artefacts. This provided the most accurate and stable numerical solutions for retrieving. The outcome of the system showed that it more effective in removing Poisson noise, compared to several existing methods. The properties of the images are also shown and proves 
the convergences of image slices. The computational time of the proposed method is high and PSNR value for some image is less.

L. Tang, Z. Fang, C. Xiang and S. Chen [23] used the multi-scale variational decomposition for the image selective restoration. The single parameter of variational decomposition theoretically analyzed the relationship between the parameter and scale of the image features. The fixed scale parameter was replaced by the single parameter with a varying sequence in the single parameter decomposition. An alternative and iterative algorithm of chambolle's projection algorithm was used to solve the multiscale variational decomposition models. The proposed method was tested and showed better performance in terms of PSNR and SSIM. The computational cost of this method is high and optimization is required for it.

Zouhair Mbarki, et al. [24] presented a new rapid and blind algorithm for image restoration technique, which doesn't require prior knowledge about the noise distribution. The degraded image deconvoluted in Fourier space by Wiener filtering and smoothed by the wave atom transform. The quality of the reconstructed image was high and computation time was low. This method is not much efficient for deblurring process because the wiener filter considers only a few features.

Most of the research are not efficient in obtaining the high quantity of restored images from the degraded images. This lack of efficiency has been overcome by using hybrid technique of NLGBT and wave atoms technique.

\section{Proposed method}

Digital image processing, such as thermoacoustic computerized tomograph (T-CT), charge-coupled device sensor (CCD-S), etc., plays an important role in the major of engineering and medicine. To process the multi variant image, it requires to obtain the indirect information (measure) of the unknown images that are used to constructing some maps and restore the digital image from the measure. In this research, NLGBT and wave atoms techniques used to restore the degraded image. The input image is degraded with Gaussian blur and then noise is added to the image for evaluating the proposed NLGBT and wave atom technique. Similar patches transformed into GBT in order to learn the basic vectors from the correlation graph and wave atoms from the graph with threshold coefficient and smoothen the transform function. Then image is restored and then evaluated using PSNR and SSIM

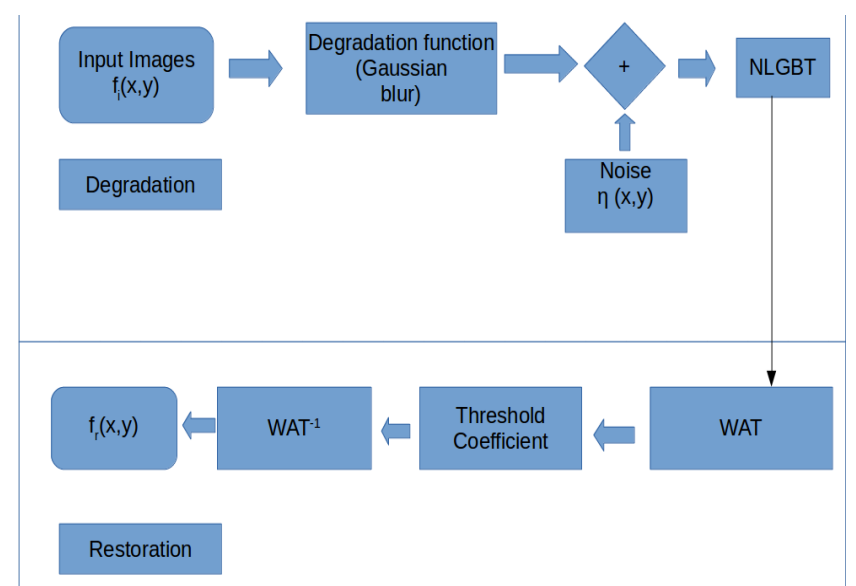

Figure.1 Architecture for the hybrid method

value. The architecture of the proposed image restoration technique is shown in the Fig. (1). The input image is denoted as $f_{i}(x, y)$ and restored image is denoted as $f_{r}(x, y)$, noise is represent as $\eta(x, y)$.

\subsection{Graph based transform}

The degraded noisy images use an input to the Graph Based Transform technique, that plot the graph based on the value extracted from the images.

The basic vectors learned from the plotted graph. Consider the images follows a zero-mean, GMRF model. The covariance matrix $\Sigma=Q-1$ are measured from the input images, as shown in Eq. (1), where $Q$ is the precision matrix. Thus the KarhunenLoève transform or the eigenvectors matrix $\Phi$ or $\Sigma$ decorrelates $x$.

$$
\Sigma \Phi=\Phi \Lambda
$$

Where $\Lambda=\operatorname{DIAG}\left(\lambda_{0, \ldots}, \lambda_{n}\right)$ is the diagonal matrix of Eigen values for $\Sigma$ as in the Eq. (2).

$$
Q \Phi=\Sigma^{-1} \Phi=\left(\Phi \Lambda \Phi^{T}\right)^{-1} \Phi \quad=\Phi \Lambda^{-1}
$$

The eigenvector matrix of precision matrix $Q$ by the given zero-mean GRMF is the optimal decorrelation linear transform. The value of GRMF is plotted as the graph and this is called as the graph transform. From the actual image statistics, the precision matrix $Q$ is constructed and so GMRF is a very generic model. In the image prior model, Laplacian matrix $\mathrm{L}$ of the graph $\mathrm{G}$ is widely used for the various kind of applications. The adjacency matrix $A$ can be used to define the Laplacian matrix $L$, where adjacency matrix $A(i, j)=A(j, i)=1$. The $i$ and $j$ in the matrix are the immediate neighbor connected by the edges and if $I$ and $J$ are connected then $A(i, j)=A(j, i)=0$. The particle correlative between the node can be used to present the 
weighted graph, then the specific weight is used to denote the $A$. The diagonal matrix is measured using the degree matrix $D$ from the Graph $G$, whereas $i^{t h}$ element defines the non-zero element in the entire row of $A$. The Laplacian of the graph is measured in the Eq. (3).

$$
L=D-A \text {. }
$$

The precision matrix $Q$ of a GMRF is measured in the Eq. (4).

$$
Q=\delta L
$$

Where scale factor is $\delta$ and if the images use GMRF model, the eigenvector of the Laplacian matrix $\mathrm{L}$ gives the optimal linear decorrelation transform. Edge Adaptive transform (EAT) is the transform design, which is recently proposed in the depth map coding. The depth map coding converts the graph into image pixels and its edges are assigned as zero. Above analysis shows that under GMRF model, EAT is the optimum transform to decorrelate the signal.

\subsubsection{Group sparsity}

The feature selection technique is explained in this section using group sparsity properties for the image restoration task.

Notations: Consider the data of $\left(x_{i}, y_{i}\right)$, where $x_{i}=\left(x_{i 1}, \ldots, x_{i p}\right)$ and $y_{i}$ are the regressors and the response for the observation. $X$ contains $n \times p$ feature matrix along with the row $j$ of $X$ and the element in the row as $X_{j}$. The sparse linear combination of the features with the stochastic noise is applied to measure $Y=\left(y_{1}, \ldots, y_{n}\right) \in R$.

Vector $\epsilon \in R^{n}: Y=X w+\epsilon$ contains the weight vector $\mathrm{w}$ and helps in the feature selection. The least square estimate is widely used as loss function to calculate the weight in the regression problem. This is also said to be the minimizer of the residual sum of squared errors in the Eq. (5).

$$
(\hat{w})=\arg _{w \in R} \min ^{p}\left\|X_{w}-Y\right\|_{2}^{2}
$$

Annotation: The similarity of the image pair is measured using the regressor $y_{i}$ and the image annotation, whereas the value $y_{i}=1$ denotes the sample is positive and the images in the $i^{\text {th }}$ pair are similar and the value $y_{i}=2$ denotes that the pair are negative and dissimilar. The keywords are included in the objective function and the similarity measured are considered as similarity of the pair. This is also said to be positive keywords that share the keyword, whereas negative keywords don't share. The pair quality helps to provide the high efficiency in the performance and usually it doesn't have the ground truth. The available $x_{i}$ have the vector difference between the features of the $i^{\text {th }}$ samples (a pair of images). If the $w_{j}=0$, then the vector $x_{i}$ is not presented in the $j^{\text {th }}$ element. The $j^{\text {th }}$ element is prunted directly depend on the prunted features and weight, the similarity between the training and testing are measured. The keyword of the image with the highest similarity is annotated to the test image and high outcomes are produced by the higher similarity. The learning method incorporating the keywords, helps to have higher performance than the JEC technique. The JEC techniques consider the visual feature distance for identifying the similarity in the test and train images.

Sparsity prior: The analytical solution of the $w$ is represented as $\left(X^{T} X\right)^{-1} X^{T} Y$ and the model is unstable due to the singular matrix $X^{T} X$. Due to the correlation of variable or the $p$ can be larger than $n$, the matrix is made singular. Generally, this problem is alleviated using the regularization method, as shown in the Eq. (6).

$$
\begin{aligned}
& \widehat{\mathrm{w}}=\operatorname{argbinonminw} \in R^{p}\left[\frac{1}{n}\left\|X_{w}-Y\right\|_{2}^{2}+\right. \\
& \left.\lambda\|w\|_{q}\right]
\end{aligned}
$$

Where $q=2$ denotes the regularization of $L^{2}$ and $q=1$ denotes $L^{1}$ regularization, and $\lambda\|w\|^{2}$ as prior in which $w$ should not be too large. The $L^{2}$ regularization doesn't induce the parsimony, as the stability of the system increases since $L^{2}$ doesn't incorporate sparsity. If the irrelevant features are present in the $X$, then the sparse prior generates the weight that provides high performance compared to those with the $L^{2}$ constraints. A sparsity prior is incorporated in the $\lambda\|w\|^{1}$ and in the sparse of $L^{0}$ regularization, the solution is present in some undetermined system. The sparse has been generated by using this prior alone, which further develop the effectiveness of the system.

Clustering prior: The features are selected using the clustering prior in the image restoration method and histogram of HSV and RGB are similar, those are clustered as same feature. The feature group structure is utilized for features selection in the method.

Thus the problem is reformulated as in Eq. (7). 


$$
\begin{gathered}
\widehat{w}=\underset{\operatorname{argmin} w}{ } \in R^{p}\left[\frac{1}{n}\left\|X_{w}-Y\right\|_{2}^{2}+\right. \\
\left.\lambda \sum_{j=1}^{m}\left\|w_{G_{j}}\right\|_{2}\right]
\end{gathered}
$$

The features are separated in the $m$ disjoint groups such as $G_{1}, G_{2}, \ldots, G_{m}$ and the group of weights are denoted as $w_{g_{j}}$ related to the group feature $G_{j}$. The groups are usually defined as an different types of $m$ features such as RGB, Haar, etc. $\lambda \sum_{j=1}^{m}\left\|w_{G_{j}}\right\|_{2}$ is the combination of both $L^{1}$ and $L^{2}$ norms. $L^{2}$ provides the weight in the same group and $L^{1}$ is the sum of the outputs of the groups. This technique is used to obtain more accurate weight and this includes the sparsity in the method. Using the leverage of group sparsity as regularization weight, $w$ is generated during the training stages and in the testing phase, similarity between the training and testing images are measured. After the calculation of Graph Based Transform, non-local value has been chosen by using the Non-local GBT technique.

\subsection{Non-local GBT}

The group sparsity in the depth maps of GBT domain are analysed in a non-local fashion due to its desirable characteristics of piecewise smoothness. The main aim of the graphical technique is to plot the image features, which shows the structure of the principal manifold. The first representation in the domain is the common geometry of the group by measuring the average of similar patches and this gives the average statistics. Then the graph of the GBT is plotted based on the similarity graph built by its average value. The pairwise similarities are measured from the adjacent pixels from the depth values of the graph. The dictionary $U$ is learned by the GBT, which is based on the mean patch $\bar{y}$ of the group:

$$
U=U(\bar{y})
$$

Once the GBT is constructed based on the average value of the similar patches, the GBT dictionary learning is introduced in the Eq. (8). The non-similarity geometry from the graph are used to reconstruct each patch in the group. By allowing different sparse representation in the GBT domain preserves the details of the image. Complicated dictionary process reduces the performance of the system and in the NLGBT approach, the dictionary has been effectively learned from the similar patches. The group sparsity has been exploited by nonlocal GBT learning algorithm from the Eq. (8) and this is based on the local piecewise smoothness prior and nonlocal self-similarity prior.

\subsubsection{Algorithm for nonlocal GBT}

\subsubsection{Patch clustering}

The first search of K-nearest-neighbours $(\mathrm{kNN})$ with exemplar patch is used for clustering features from the given patches. This is stacked as column to create a data matrix Y. Block-matching and Euclidean distance are the exemplar patch used by the BM3D technique as well as the $\mathrm{k}$ most similar patches to the exemplar patch.

\subsubsection{GBT dictionary learning}

The common structures elaborated by the GBT technique are used to learn the similar patches [25]. The local neighbourhood correlations between the pixels are measured as average patch used to identify the similar patches. There are some similarity graphs available such as a -neighbourhood graph, fully connected graph, and k-nearest neighbour graph [26]. The undirected weighted graph is plotted based on the average patch as a node and connect adjacent pixels $(i, j)$ with edge weight in the Eq. (9).

$$
w_{i, j}=e^{\frac{-\left\|y_{i}-y_{j}\right\|^{2}}{\sigma_{w}^{2}}}
$$

Where the squared intensity difference between the pixel $i$ and $j$ similarity is the $\left\|y_{i}-y_{j}\right\|^{2}$ is measured. The parameter $\sigma_{w}$ control the sensitivity of the similarity measure between the noise and the range of the intensity difference in the patch. The noise variance parameter is set as $20 \%$ and the maximum intensity difference in the patch is calculated. The weighted similarity graph and the GBT basic functions are calculated from the Eigendecomposition, these values are used to measure the corresponding Laplacian matrix. The weighted GBT usually provides sparse representation of the average patch, that measure the subtle inter-pixel correlations in the patch.

\subsubsection{Image update}

The inverse GBT with sparsified transform coefficient are used to reconstruct all the patches and various estimates are learned from the overlapped patches of each patch, updated by the depth map using weighted averaging. The weight and the order of sparsified coefficient matrix should be inversely proportional. Hence, the higher sparse patches get the highest priority during weighting average. The 
weight is set as $c_{j}=1-\left(r_{j} / n\right)$ for the $j^{\text {th }}$ cluster, where the rank is denoted as $r_{j}$ and patch size is $n$.

\subsubsection{Iterative regularization}

The filtered noise back is used in the iterative regularization [27] to denoise the image at each iteration to improve the quality of the image based on the previous estimation using Eq. (10).

$$
\hat{y}^{(k+1)}=\hat{y}^{(k)}+\delta\left(y-y^{(k)}\right),
$$

Where the input noisy depth map is denoted as $y$, the denoised version at the $k^{\text {th }}$ iteration is represented as $\hat{y}(k)$ and the relaxation parameter is denoted as $\delta$.

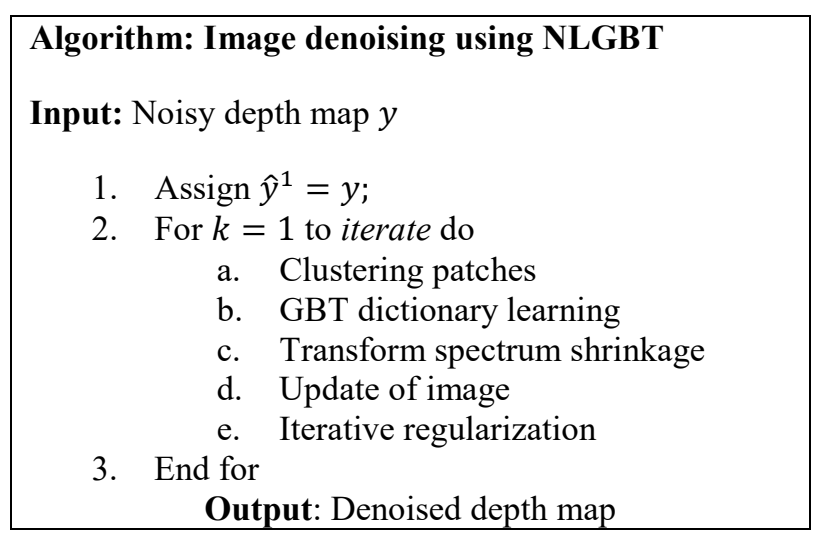

The non-local value is selected from the Graph Based Transform and this is fed to the wave atoms to obtain the high quality images.

\subsection{Wave atom}

The execution of the wave atom and digital wave atom techniques are applied to the input images using basic vectors [28], [29]. The wave atom is explained in the two dimensions and write $x=$ $\left(x_{1}, x_{2}\right)$ and this is not essential. The conventional model for 2D Fourier transform is given in Eq. (11).

$$
\begin{aligned}
& f(w)=\int e^{i x \omega} f(x) d x, \\
& f(x)=\frac{1}{(2 \pi)^{2}} \int e^{i x \cdot \omega} \operatorname{hat} f(\omega) d \omega
\end{aligned}
$$

Wave atoms are written as $\varphi_{\mu}(x)$, with subscript $\mu=(j, m, n)=\left(j, m_{1}, m_{2}, n_{1}, n_{2}\right)$ and all five qualities $j, m_{1}, m_{2}, n_{1}, n_{2}$ are integer-valued and index point $\left(x_{\mu}, \omega_{\mu}\right)$ in phase-space, as in the Eq. (12).

$$
x_{\mu}=2^{-j} n, \omega_{\mu}=\pi 2^{j} m,
$$

$$
C_{1} 2^{j} \leq \underset{i=1,2}{\max } \leq C_{2} 2^{j}
$$

Where two positive constants $C_{1}, C_{2}$ are not specified for convenience, but those values will vary based up on the implementation. The $x_{\mu}$ is the position vector that is the centre of $\varphi_{\mu}(x)$ and the wave vector $\omega_{\mu}$, calculate the centers of both bumps of $\hat{\varphi}_{\mu}(\omega)$ as $\pm \omega \mu$. The value of $m$ need to be minimized to $m_{2}>0$ (or $m_{2}=0$ and $m_{1}>0$ ) to account for the central symmetry of the Fourier transform of real-valued functions about the origin in $\omega$. The restriction is provided for the $n$ and $j$, but not in the description of a frame $L^{2}\left(R^{2}\right)$.

The localization condition around the phasespace point $\left(x_{\mu}, \omega_{\mu}\right)$ followed in the wave atom for image reconstruction.

\subsubsection{Digital wave atoms}

Wave atoms are the tensor product from a special kind of 1D wave packets. In the continuous frequency, defining the wave atom as follows in the Eq. (13).

$$
\begin{gathered}
\hat{\psi}_{m}^{0}(w)=e^{-i w / 2}\left[e ^ { i \alpha _ { m } } g \left(\epsilon_{m}(w-\right.\right. \\
\left.\left.\left.\pi\left(m+\frac{1}{2}\right)\right)\right)+e^{-i \alpha_{m}} g\left(\epsilon_{m+1}\left(w+\pi\left(m+\frac{1}{2}\right)\right)\right)\right]
\end{gathered}
$$

Where $\alpha_{m}=\pi 2(m+12)$ and $\epsilon_{m}=(-1) m$. The $\mathrm{g}$ is the real-valued, convenient to support $C^{\infty}$ bump function are shown in Eq. (14)

$$
\sum_{m}\left|\psi_{m}^{0}(w)\right|^{2}=1
$$

Translates the Eq. (14) on the basis of an orthonormal form and this provides the Gabor, tiling, uniform of the frequency axis.

Template $\psi_{m}^{0}$ dilated by the Villemoes's construction and generates wavelet packet-like orthobases that have high frequency localization in the non-Gabor form. The index scale has been incorporated with the subscript $j$, is provided in Eq. (15).

$$
\psi_{m, n}^{j}(x)=\psi_{m}^{j}\left(x-2^{-j} n\right)=2^{j / 2} \psi_{m}^{0}\left(2^{j} x-n\right)
$$

The coefficients associated to $\psi_{m, n}^{j}(x)$ usually denoted as $c_{j, m, n}$. The wavelet packet of tree points 
are represented in $(j, m)$ and the depth at the point is $J-j$. In that function, $J$ is the maximum depth and number of nodes on the left at the same depth is denoted as $m$ [24]. This is the common indexing method for the wavelet packets and $j$ within Fourier uncertainty principle. From the given atom $\psi_{m, n}^{j}(x)$ is center at space $(x, \omega)$ in the Eq. (16).

$$
x_{j, n}=2^{-j} n, \quad w_{j, m}=\pi 2^{j} m
$$

The wave atom is designed and the subscript $m$ is set, that is comparable to $2^{j}$ in the absolute value. The parabolic scaling law is followed, the window width in the frequency $\left(2^{j} 2 \pi\right)$ in the asymptotically, up to constant, and the square root of the distance to the origin $(\sim 22 j)$. The essential width of the support in $x\left(\sim 2^{-j}\right)$ is provide the square root of the wavelength in the dual fashion inside the envelope $\left(\sim 2^{-2 j}\right)$.

The parabolic scaling law is followed in this method: the window

The Hilbert-transformation is used to obtain a second orthonormal basis, is shown in Eq. (17). The Hilbert-transformation is based on the real variable.

$$
\begin{aligned}
& H \hat{\psi}_{m}^{0}=e^{-i w / 2}\left[( - i ) e ^ { i \alpha _ { m } } g \left(\epsilon_{m}(w-\right.\right. \\
& \pi(m+1 / 2)))+i e^{-i \alpha_{m}} g\left(\epsilon_{m+1}(w+\right. \\
& \pi(m+1 / 2)))]
\end{aligned}
$$

The other values of the $j$ are adequately dilated and the coefficients of the Hilbert-transformation is denoted by $c_{j, m, n}^{H}$.

There are three ways available to combine 1D wave atoms in order to define $2 \mathrm{D}$ wave atoms:

\section{An orthonormal basis:}

The tensor products are considering individually in a "nonstandard" manner for creating the 4 bumps orthonormal basis function in the frequency plane with only one dilation parameter $\mathrm{j}$. So, let $\mu=$ $(j, m, k)$, where $m=\left(m_{1}, m_{2}\right)$ and $n=\left(n_{1}, n_{2}\right)$, are expressed in Eq. (18). The $m$ denotes the five qualities.

$$
\begin{aligned}
& \varphi_{\mu}^{+}\left(x_{1}, x_{2}\right)=\psi_{m_{1}}^{j}\left(x_{1}-2^{-j} n_{1}\right) \psi_{m_{2}}^{j}\left(x_{2}-\right. \\
& \left.2^{-j} n_{2}\right)
\end{aligned}
$$

The Fourier transform is also separable, namely $\hat{\psi}_{\mu}^{+}\left(w_{1}, w_{2}\right)=$
$\psi_{m_{1}}^{j}\left(w_{1}\right) e^{-i 2^{j} n_{1} w_{1}} \hat{\psi}_{m_{2}}^{j}\left(w_{2}\right) e^{-i 2^{j} n_{2} w_{2}}$.

The Hilbert-transformed wavelet packets can be used to define a dual orthonormal basis in the Eq. (19).

$$
\begin{aligned}
& \overline{\varphi_{\mu}}\left(x_{1}, x_{2}\right) \equiv H \psi_{m_{1}}^{j}\left(x_{1}-2^{-j} n_{1}\right) H \psi_{m_{2}}^{j}\left(x_{2}-\right. \\
& \left.2^{-j} n_{2}\right)
\end{aligned}
$$

2. A tight frame with redundancy:

The main drawback of the defined function is the $x$ space oscillate in the two direction instead of one. This issue is solved by combining the primal and dual basis. The recombination is in the Eq. (20).

$$
\begin{array}{cc}
\frac{\varphi_{\mu}}{2}, & \frac{\varphi_{\bar{\mu}}}{2}, \\
\varphi_{\mu}^{(1)}=\varphi_{\mu}^{+}+ & \varphi_{\mu}^{(2)}=\varphi_{\mu}^{+}-
\end{array}
$$

Basic function with the two bumps are provided and purely directional wave atom is computed. Together, $\varphi_{\mu}^{(1)}$ and $\varphi_{\mu}^{(2)}$ form the wave atom frame and may be denoted jointly as $\varphi_{\mu}$.

3. A tight frame redundancy four:

The tight frame with two redundancies can be used for the numerical analysis of the data compression. For the statistical estimation, translation invariance matters are important to differentiate the design. Separating the bumps in the frequency plane helps to provide the shift-invariance of the subspaces for fixed $j$ and $m$ with quadrant indicators. This creates the complex-valued basis functions. The phase factors are not used and orthobasis is constructed for quadruple redundancy.

The wave atoms of frequency tiling are shown in the Eq. (21). In contrast to curvelets, about $O\left(2^{j} / 2\right)$ wave atom window has to be cover a SSD wedge at scale $j$. The frequency sampling and wrapping in curvelets are used in the discretization of wave atoms. The following equation is defined for the $1 \mathrm{D}$ wave atoms.

$$
c_{j, m, n}^{D}=\sum_{w \in 2 \pi Z} e^{i 2^{j} n k} \overline{\hat{\psi}_{m}^{j}(w)} \hat{f}(w)
$$

Modulo aliasing complicate the basic functions at the smallest scale, let $\omega=2 \pi(-N / 2+$ $1: 1: N / 2)$ in the Eq. (21). The wrapping process is a periodization of the sample of frequency that helps to provide the Eq. (22). 


$$
\begin{aligned}
& \sum_{0 \leq j \leq J} O\left(j 2^{2}\right)=O\left(J 2^{2 J}\right)=O(N \log N), \\
& \text { With } N=2^{2 J}
\end{aligned}
$$

The expressions denoted for the 2D simple tensor products and flowchart are approximately related to the curvelets and yields an $O(N \log N)$ algorithm in $1 \mathrm{D}-O(N 2 \log N)$ in $2 \mathrm{D}$. All three wave atom transform variant are isometrics and essential for the same complexity by the adjoint.

\section{Experimental study}

The image restoration process has been carried out with NLGBT and wave atoms technique and it is evaluated in terms of PSNR and SSIM. The experiment was tested in MATLAB R2018a in the processor of Intel i3 core with RAM of 4GB. The inbuild MATLAB images were taken for the experiment analysis.

The parameter that involves in measuring the quality of the restored image using the NLGBT and wave atom are PSNR, Normalized Cross Correlation (NCC) and SSIM. The formula to calculate the PSNR, SSIM, NCC, MSSIM and NMSE are given as follows in the Eq. (23-27).

$$
\begin{aligned}
& \text { PSNR }=10 \log \left(\frac{d}{M S E}\right) \\
& \text { With MSE }=\frac{1}{M N}([f(x, y)-r(x, y)]) \\
& \operatorname{SSIM}(x, y)=\frac{\left(2 \mu_{x} \mu_{y}+c_{1}\right)\left(2 \sigma_{x y}+c_{2}\right)}{\left(\mu_{x}^{2}+\mu_{y}^{2}+c_{1}\right)\left(\sigma_{x}^{2}+\sigma_{y}^{2}+c_{2}\right)} \\
& \operatorname{NCC}=\frac{\sum_{i=1}^{M} \sum_{j=1}^{N} x(i, j) X_{y}(i, j)}{\sum_{i=1}^{M} \sum_{j=1}^{N}\left(x(i, j)^{2}\right)} \\
& M S S I M=\frac{1}{P} \sum_{j=1}^{P} S S I M_{j} \\
& N M S E=\frac{\sum_{i=1}^{m} \sum_{j=1}^{n}\left(I^{\prime}(i, j)-I(i, j)\right)^{2}}{\sum_{i=1}^{m} \sum_{j=1}^{n} I^{2}(i, j)}
\end{aligned}
$$

With $\mu_{x}$ is the $x$ average, $\mu_{y}$ is the $y$ average, $\sigma_{x}^{2}$ is the $x$ variance, $\sigma_{y}^{2}$ is the $y$ variance, $\sigma_{x y}$ is the $x$ and $y$ covariance, $c_{1}=\left(k_{1} L\right)^{2}$ and $c_{2}=\left(k_{2} L\right)^{2}$ stabilize the division with weak denominator and $\mathrm{L}$ is the dynamic range of the pixel-value $k_{1}=0.01$ and $k_{2}=0.03$ by default. The last criterion depends on the edge detection from restored images with Canny operator or 0.1 threshold.

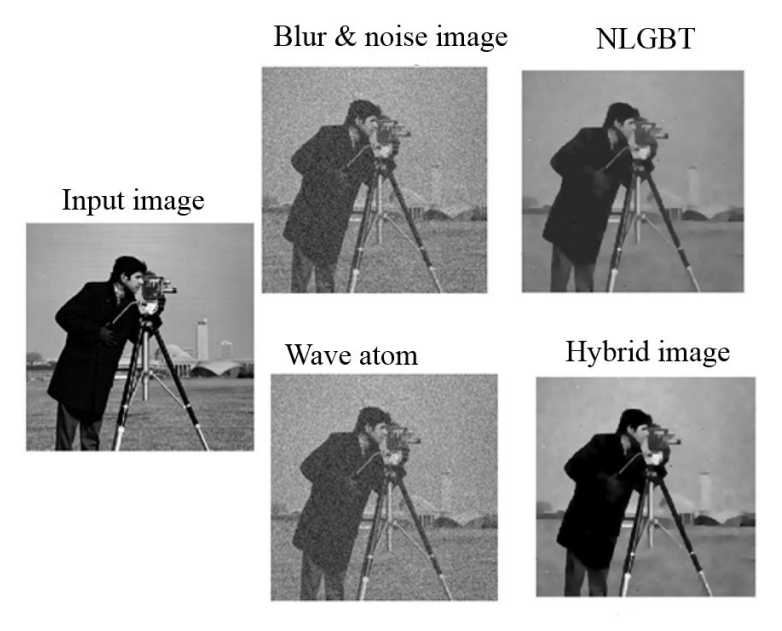

Figure.2 Cameraman images: (a) original image, (b)

Gaussian blur and noise image, (c) NLGBT images, (d) wave atoms images, and (e) hybrid images

\section{Experimental result}

Image restoration is the basic function in the image processing technique and it is highly used in the many field especially in the medical domain. The visual details of the images are highly important for this application to diagnosis the diseases. The stateof-art method has the considerable performance in presenting the high quality restored image and still in the need of robustness. Here, the NLGBT and wave atom technique is proposed to create the image with less noise and low blur. The quality of the images is evaluated in terms of different parameter. The images were given to the NLGBT to learn the basic vector from the plotted graph transform and wave atom technique was used to smoothen the image. This method was compared with wiener filter technique and the wave atoms technique [29]. Gaussian blur was applied to the images in the size of $21 \times 21$ generated in the MATLAB built-in function and then Gaussian noise was added with $\mathrm{BSNR}=30 \mathrm{~dB}$.

The cameraman images are degraded using the technique of Gaussian blur and Gaussian noise and attempt has been made to reconstruct the images with high quality. The degraded cameraman image is shown in the Fig. 2 (b). Then the various techniques have been applied to the blurred image to restore the image with less noise. The wave atom and NLGBT is applied individually in the images. Figs. 2 (c) and 2 (d) present the restored image of the both methods. The proposed hybrid method is applied to the degraded image and resultant image is shown in the Fig. 2 (e). The visual observation of the Fig. 2 shows the hybrid techniques have the high quality compared to the other techniques. The images 
Table 1. PSNR value

\begin{tabular}{|c|c|c|c|c|c|c|}
\hline & Wiener filter & Wavelet & $\begin{array}{c}\text { Wiener-Wave } \\
\text { atoms [24] }\end{array}$ & $\begin{array}{c}\text { Wave } \\
\text { atoms }\end{array}$ & NLGBT & Hybrid \\
\hline House & 17.29 & 18.90 & 20.08 & 18.57 & 28.52 & 32.5 \\
\hline Lena & 18.23 & 20.44 & 21.24 & 18.81 & $27 . .8$ & 29.7 \\
\hline Cameraman & 21.24 & 22.96 & 24.03 & 18.52 & 25.69 & 30.1 \\
\hline
\end{tabular}

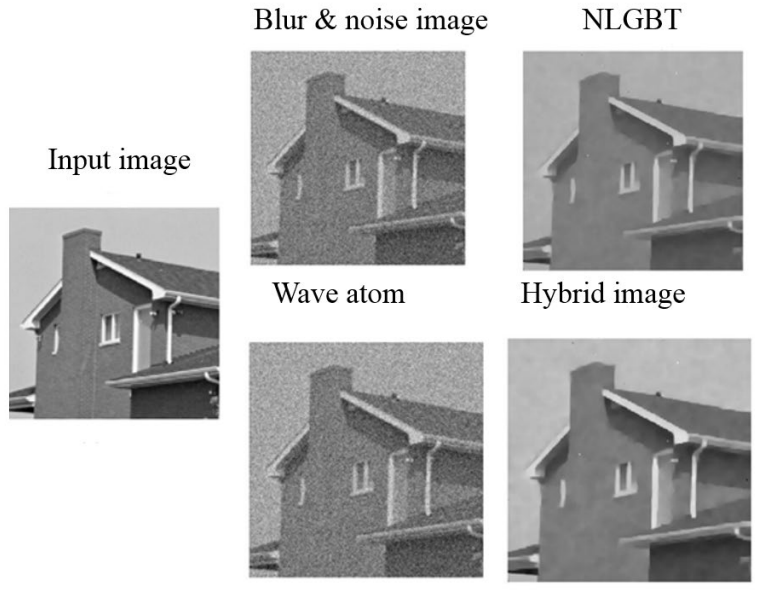

Figure. 3 House images: (a) input images, (b) Gaussian blur and noise image, (c) NLGBT images, (d) wave atom images, and (e) hybrid images
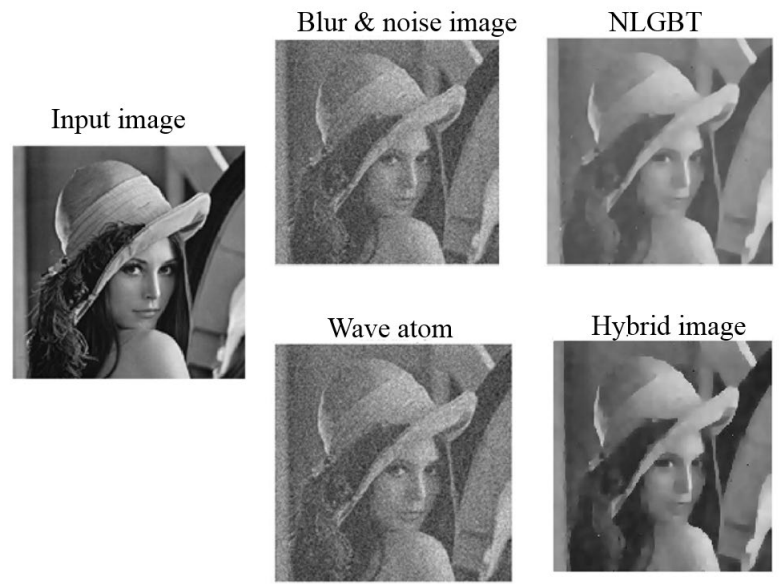

Figure.4 Lena images: (a) input images, (b) Gaussian blur and noise image, (c) NLGBT images, (d) wave atom images, and (e) hybrid images

reconstructed from the various method including hybrid technique are evaluated in terms of different parameter to investigate the effectiveness of the Hybrid method.

Likewise, house images are degraded and restored using the NLGBT technique, Wave atom technique and proposed hybrid technique. The NLGBT and wave atom technique restored images are shown in the Fig. 3.(c) \& (d) respectively. The images restored using the Hybrid method are shown in the Fig. 3(e). The visual observation of the Fig. 3 shows that Hybrid method has the higher performance compared to the other techniques.

The various method involves the image reconstruction are evaluated using the in-built MATLAB images. The cameraman, House and Lena images were tested with NLGBT and Wave atom images, and the outcome images is shown in the Fig. (2), (3) \& (4). The proposed hybrid method shows its efficiency in the visual details.

The proposed hybrid technique is applied to the Gaussian blur images and reconstructed less noise images. The reconstructed image was measured on the various parameter, which is compared with other technique such as Gaussian blur, NLGBT images, wave atom images and proposed technique images, those are shown in the Fig. (2), (3) \& (4). The house images and cameraman images used in the size of $(21 \times 21)$ and Lena images were taken in $(512 \times 512)$. The outcome showed that the wave atoms images restored the images with less PSNR value and proposed hybrid technique of NLGBT and wave atoms achieves better restored images.

PSNR value is calculated for 3 images and it is compared with other existing method in Table 1, which shows the effectiveness of the proposed NLGBT-wave atom method. Instead of smoothing of images like wiener-wave atom [24], the proposed hybrid method involves in smoothing of basic vectors of the image. This helps to increase the performance and minimize the computation time. The state-of-the-art method achieved PSNR of 20.08 $\mathrm{dB}$ for House images, while Hybrid method achieved up to $29.02 \mathrm{~dB}$. Other images include Lena, and cameraman PSNR value is higher in the Hybrid method. NLGBT also achieved the considerable PSNR in all 3 images. The hybrid method has the highest PSNR value in the house image. The existing method also evaluated the House, Lena images in the size of $21 \times 21$ pixel and Lena image in $512 \times 512$ pixel. 
PSNR

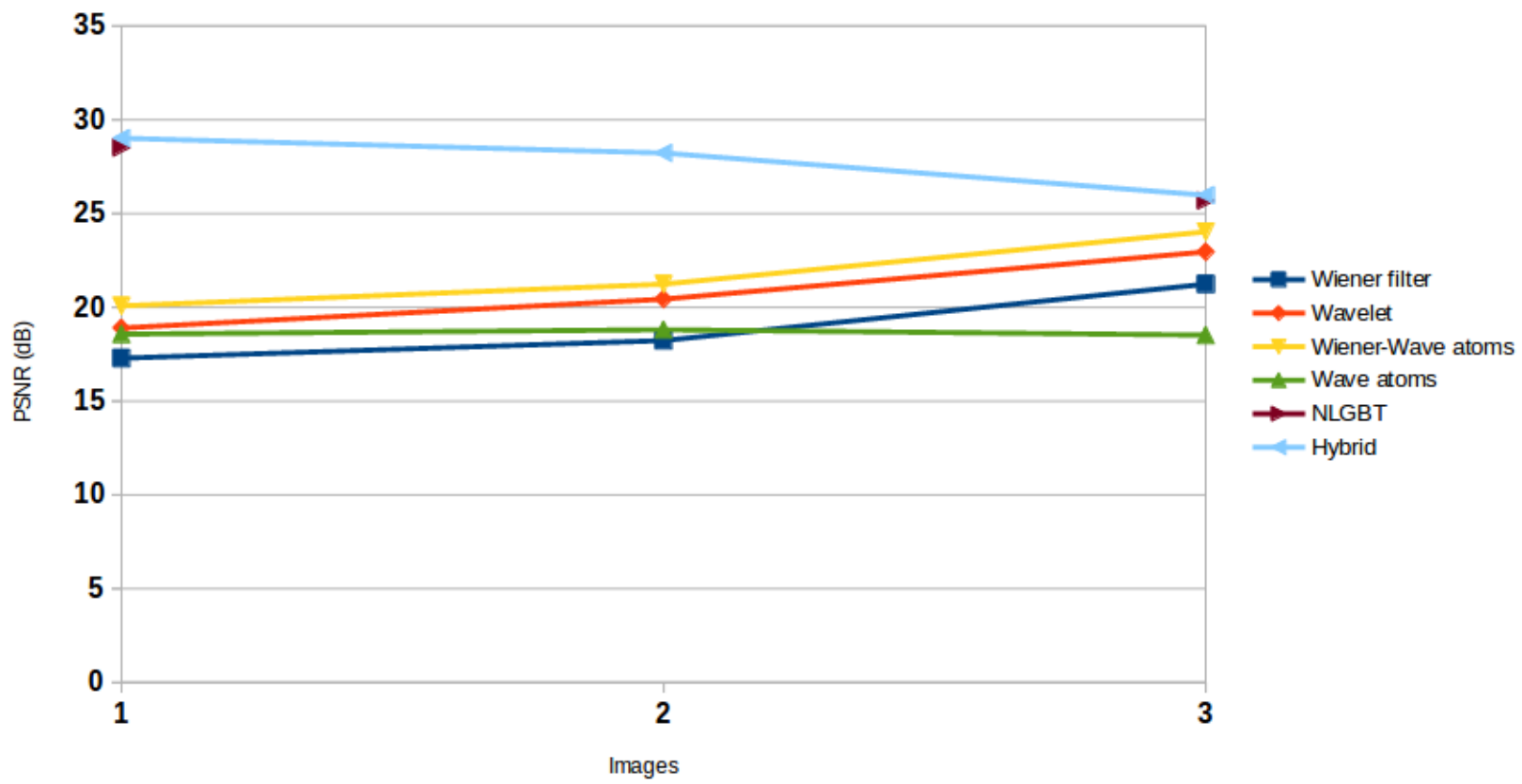

Figure.5 PSNR comparison

Table 2. PSNR comparison for different methods

\begin{tabular}{|c|c|c|c|}
\hline & House & Lena & Cameraman \\
\hline BM3D & 29.7 & 27.4 & 27.5 \\
\hline NLM & 28.5 & 26.2 & 26.4 \\
\hline BF & 24.2 & 24.1 & 24.3 \\
\hline Hybrid & 32.5 & 29.7 & 30.1 \\
\hline
\end{tabular}

Table 3. PSNR measure in Gaussian blur $\sigma=2$

$(21 \times 21)$ Gaussian blur with $\sigma=2$ and Gaussian noise with BSNR $=20(\mathrm{~dB})$

\begin{tabular}{|c|c|c|c|c|c|}
\hline Images & $\begin{array}{c}\text { NBID } \\
\text { method(d } \\
\text { B) [24] }\end{array}$ & $\begin{array}{c}\text { Blind } \\
\text { Algorith } \\
\mathbf{m}(\mathbf{d B}) \\
{[\mathbf{2 4}]}\end{array}$ & $\begin{array}{c}\text { Wav } \\
\mathbf{e} \\
\text { atom } \\
\mathbf{s}\end{array}$ & $\begin{array}{c}\text { NLGB } \\
\mathbf{T}\end{array}$ & $\begin{array}{c}\text { Hybri } \\
\mathbf{d}\end{array}$ \\
\hline $\begin{array}{c}\text { Cameram } \\
\text { an }\end{array}$ & 25.20 & 25.55 & 19.27 & 21.75 & 21.97 \\
\hline Lena & 27.12 & 28.35 & 19.84 & 22.15 & 22.85 \\
\hline Barbara & 22.28 & 22.63 & $\begin{array}{c}21.05 \\
5\end{array}$ & 26.44 & 26.48 \\
\hline Baboon & 19.55 & 19.68 & 19.07 & 21.4 & 22.09 \\
\hline
\end{tabular}

The comparison of the various method in terms of PSNR for 3 images presented in the Fig. (5) and this clearly shows that the Hybrid method has higher PSNR value. NLGBT method achieved the second higher PSNR value. Hybrid technique achieved higher PSNR compared to the other methods. Wiener filter method used in the wave atoms to get considerable PSNR value and NLGBT nearly equal to hybrid technique in PSNR value.

The image restoration has been carried out for the different method such as Block matching 3D (BM3D) [30], Non-local means denoising (NLM) [31], and Bilateral Filter (BF) [32]. The three images such as house, Lena and Cameraman are added with noise $(\sigma=20)$ and these methods were simulated with same environment. The output obtained from these methods are compared with Hybrid method, as shown in the Table 2. The Hybrid method has the higher PSNR value compared to the other existing method. The Hybrid method has the PSNR value of $32.5 \mathrm{~dB}$, while BM3D has the PSNR value of 29.7 $\mathrm{dB}$. The hybrid method has the higher efficiency in the image restoration compared to the other existing method.

The Table 3 shows the image with Gaussian blur of variance $(\sigma=2)$ and Gaussian noise of BSNR $=20$ $\mathrm{dB}$ in the size of $21 \times 21$ pixel. While the Gaussian blur variance is $\sigma=2$ in the images, proposed system achieved higher PSNR for 2 images, namely Barbara and Baboon images and have less PSNR for other 2 images. The cameraman and Lena images attained lower PSNR than other images. The image restoration technique evaluated in the colour image and the outcome is discussed.

The colour images are used in the many applications like face detection, fruit quality detection and the various image restoration techniques are evaluated in the colour image. The 
Table 4. The parameter value of the restored color image

\begin{tabular}{|c|c|c|c|c|c|c|}
\hline \multirow{2}{*}{ Images } & \multicolumn{2}{|c|}{ Wave atom } & \multicolumn{2}{c|}{ NLGBT } & \multicolumn{2}{c|}{ Hybrid } \\
\cline { 2 - 7 } & PSNR & SSIM & PSNR & SSIM & PSNR & SSIM \\
\hline Barbara & 22.53 & 0.7221 & 27.18 & 0.8688 & 27.78 & 0.8878 \\
\hline Lena & 22.5464 & 0.7824 & 29.01 & 0.9122 & 29.52 & 0.9222 \\
\hline
\end{tabular}

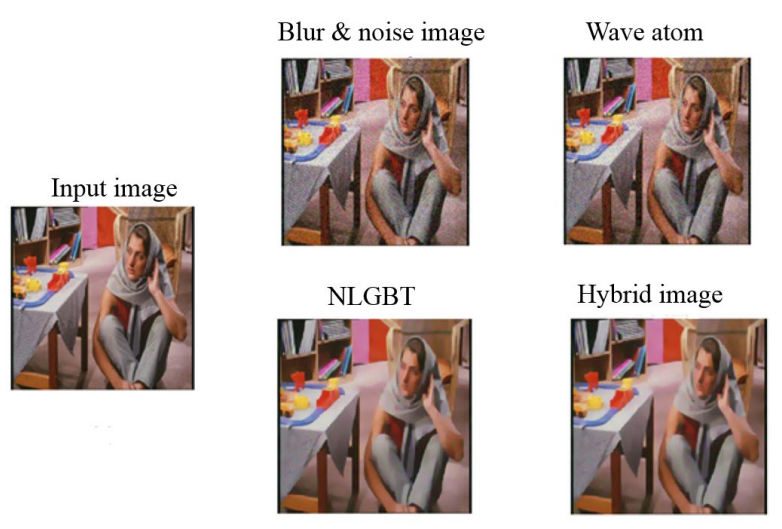

Figure.6 Barbara color image evaluation

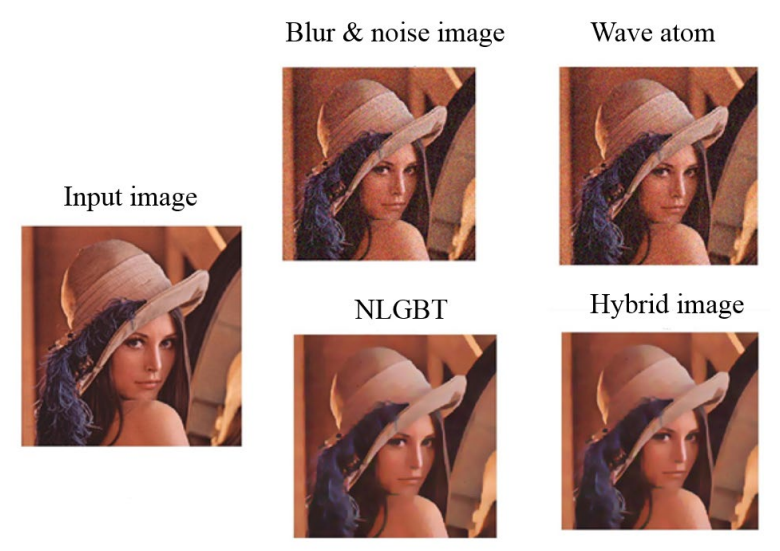

Figure.7 Lena color image evaluation in restoration

Table 5. NCC comparison

\begin{tabular}{|c|c|c|c|}
\hline Images/Methods & NBGLT & Wave atom & Hybrid \\
\hline Lena Image & 0.9802 & 0.9121 & 0.9804 \\
\hline Cameraman & 0.9882 & 0.9573 & 0.9883 \\
\hline
\end{tabular}

Barbara image are validated in the restoration process and shown in the Fig. 6. The input image is degraded and restoration process is carried out to restore the image. The Hybrid technique achieve the higher performance and wave atom method produce the image with some noise.

The Lena color image is given as the input to the restoration process and results are evaluated in the PSNR and SSIM. The result image from various techniques are shown in the Fig. 7, where wave atom techniques are observed to provide the detailed image with some noise. The hybrid technique having the higher quality compared to other two techniques. NLGBT technique reduce much noise compared to the wave atom technique with some loss of information in the image. The parameter measures from the NLGBT, wave atom technique and Hybrid technique are shown in the Table 4. SSIM parameter measures the similarity between the input image and the restored image. This helps to provide the quality of the image after applying restored technique.

The Hybrid method is measured with NCC and compared it with other existing methods, as shown in Table 5. The NCC parameter is used to measure the similarity between the images to analyse the function of displacement. The outcome image is compared with the input image for measuring the similarity. The parameter of NCC not involves in subtracting the local mean value of intensities compared to the Zero-Normalized cross correlation.

The different parameters like NMSE, MSE, and MSSIM are measured from the output of the Hybrid method and compared with existing methods of Wave atom and NLGBT that is presented in Table 6. In order to evaluate from both gray and colour images, these methods are tested in two grey images and two colour images. Barbara and Lena colour images shows lower error value (NMSE and MSE) for the Hybrid method. NLGBT method have nearly similar error value and MSSIM value to the proposed method in colour images. The Hybrid method has higher performance compared to the other existing method. The Lena and Barbara colour are tested, as it shows the Barbara image has the higher MSSIM value due to rich colour present in the Barbara image. The cameraman and house grey images are used for the analysing the performance. This shows that the proposed hybrid method has the higher performance compared to other existing method.

The computational complexity of the method is linear to the size of the input images. The computation time of the hybrid method in the MATLAB implementation takes approximately $43 \mathrm{~s}$ on the Intel-i5 process for $256 \times 256$ image. The existing method has the computation time of $92 \mathrm{~s}$ for the denoising. 
Table 6. Various Parameter value

\begin{tabular}{|c|c|c|c|c|c|c|c|c|c|}
\hline & \multicolumn{3}{|c|}{ Wave atom } & \multicolumn{3}{c|}{ NLGBT } & \multicolumn{3}{c|}{ Hybrid } \\
\hline Images & NMSE & MSE & MSSIM & NMSE & MSE & MSSIM & NMSE & MSE & MSSIM \\
\hline $\begin{array}{c}\text { Barbara } \\
\text { (Color) }\end{array}$ & 0.0091 & 362.7346 & 0.8368 & 0.0031 & $\begin{array}{c}124.506 \\
6\end{array}$ & 0.9265 & 0.003 & $\begin{array}{c}122.82 \\
06\end{array}$ & 0.9267 \\
\hline $\begin{array}{c}\text { Lena } \\
\text { (Color) }\end{array}$ & 0.0091 & 361.7884 & 0.6865 & 0.002 & 78.6889 & 0.8744 & 0.002 & $\begin{array}{c}78.080 \\
1\end{array}$ & 0.8727 \\
\hline $\begin{array}{c}\text { Camerama } \\
\text { n (Grey) }\end{array}$ & 0.009102 & 364.8472 & $\begin{array}{c}0.56660 \\
8\end{array}$ & $\begin{array}{c}0.00230 \\
7\end{array}$ & $\begin{array}{c}92.4665 \\
4\end{array}$ & $\begin{array}{c}0.75652 \\
1\end{array}$ & 0.00229 & $\begin{array}{c}91.772 \\
65\end{array}$ & $\begin{array}{c}0.76413 \\
7\end{array}$ \\
\hline $\begin{array}{c}\text { House } \\
\text { (Grey) }\end{array}$ & 0.009038 & 365.4116 & 0.5519 & $\begin{array}{c}0.00121 \\
7\end{array}$ & $\begin{array}{c}49.1976 \\
7\end{array}$ & $\begin{array}{c}0.71691 \\
8\end{array}$ & $\begin{array}{c}0.00117 \\
8\end{array}$ & $\begin{array}{c}47.637 \\
53\end{array}$ & $\begin{array}{c}0.71600 \\
9\end{array}$ \\
\hline
\end{tabular}

This can be applicable to the image restoration technique to reconstruct the images with less noise. NLBGT learns the basic vector from the plotted graph transform and Wave atom is used to smoothen the image. Through this way, the images have been restored with higher quality.

\section{Conclusion}

Restoring the quality of image from noisy image is the one of the important tasks in image processing. Earlier, many researches have been carried out on the image denoising and deblurring to improve the performance. In this research, image has been degraded and Gaussian noise is added to measure the performance of NLGBT and wave atom. The hybrid technique of NLGBT and wave atoms techniques is used to restore the images. The result showed that the performance of the proposed technique has the highest quality of restored image compared to the other existing methods. PSNR value has been achieved up to $29.02 \mathrm{~dB}$ in the house image of size $21 \times 21$ pixel in $B S N R=30 \mathrm{~dB}$. The Hybrid method has shown the higher PSNR value for most of the images. This shows the hybrid technique of NLGBT and wave atom can be applied for the accurate reconstruct of degraded images. In the future work hybrid method can be implemented to increase the quality of images.

\section{References}

[1] S. Liu, G. Wu, H. Liu, and X. Zhang, "Image restoration approach using a joint sparse representation in 3D-transform domain", Digital Signal Processing, Vol.60, pp.307-323, 2017.

[2] S. Lahmiri, "An iterative denoising system based on Wiener filtering with application to biomedical images", Optics \& Laser Technology, Vol.90, pp.128-132, 2017.

[3] C. He, C. Hu, X. Li, and W. Zhang, "A parallel primal-dual splitting method for image restoration", Information Sciences, Vol.358, pp.73-91, 2016.
[4] R. Feiz and M. Rezghi, "A splitting method for total least squares color image restoration problem", Journal of Visual Communication and Image Representation, Vol.46, pp.48-57, 2017.

[5] Z. Su, S. Zhu, X. Lv, and Y. Wan, "Image restoration using structured sparse representation with a novel parametric data-adaptive transformation matrix", Signal Processing: Image Communication, Vol. 52, pp.151-172, 2017.

[6] S. Tebini, Z. Mbarki, H. Seddik, and E.B. Braiek, "Rapid and efficient image restoration technique based on new adaptive anisotropic diffusion function", Digital Signal Processing, Vol.48, pp.201-215, 2016.

[7] Z. Wang, Z. Yao, and Q. Wang, "Improved scheme of estimating motion blur parameters for image restoration", Digital Signal Processing, Vol.65, pp.11-18, 2017.

[8] C. Wang, T. Sun, T. Wang, X. Miao, and R. Wang, "Multi-PSF fusion in image restoration of range-gated systems", Optics \& Laser Technology, Vol.103, pp.219-225, 2018.

[9] Z. Liu, L. Yu, and H. Sun, "Image restoration via Bayesian dictionary learning with nonlocal structured beta process", Journal of Visual Communication and Image Representation, Vol.52, pp.159-169, 2018.

[10] K. Sakthidasan and N.V. Nagappan, "Noise free image restoration using hybrid filter with adaptive genetic algorithm", Computers \& Electrical Engineering, Vol.54, pp.382-392, 2016.

[11] F. Sharifzadeh, G. Akbarizadeh, and Y. S. Kavian, "Ship Classification in SAR Images Using a New Hybrid CNN-MLP Classifier", Journal of the Indian Society of Remote Sensing, pp.1-12.

[12] G. Akbarizadeh, "A New Statistical-Based Kurtosis Wavelet Energy Feature for Texture Recognition of SAR Images", IEEE Trans. Geoscience and Remote Sensing, Vol.50, No.11, pp.4358-4368, 2012. 
[13] M. Modava, G. Akbarizadeh, and M. Soroosh, "Integration of spectral histogram and level set for coastline detection in SAR images", IEEE Transactions on Aerospace and Electronic Systems, 2018.

[14] G. Akbarizadeh, K. Rangzan, and M. Kabolizadeh, "Effective supervised multiplefeature learning for fused radar and optical data classification", IET Radar, Sonar \& Navigation, Vol.11, No.5, pp.768-777, 2016.

[15] M. Farbod, G. Akbarizadeh, A. Kosarian, and K. Rangzan, "Optimized fuzzy cellular automata for synthetic aperture radar image edge detection", Journal of Electronic Imaging, Vol.27, No.1, p.013030, 2018.

[16] A. Raeisi, G. Akbarizadeh, and A. Mahmoudi, "Combined method of an efficient cuckoo search algorithm and nonnegative matrix factorization of different zernike moment features for discrimination between oil spills and lookalikes in SAR Images", IEEE Journal of Selected Topics in Applied Earth Observations and Remote Sensing, Vol.99, pp.1-13, 2018.

[17] M. Norouzi, G. Akbarizadeh, and F. Eftekhar, "A hybrid feature extraction method for SAR image registration. Signal", Image and Video Processing, pp.1-8, 2018.

[18] G. Akbarizadeh and A. E. Moghaddam, "Detection of lung nodules in CT scans based on unsupervised feature learning and fuzzy inference", Journal of Medical Imaging and Health Informatics, Vol.6, No.2, pp.477-483, 2016.

[19] T. Liu, Z. Chen, S. Liu, Z. Zhang and J. Shu, "Blind image restoration with sparse priori regularization for passive millimeter-wave images", Journal of Visual Communication and Image Representation, Vol.40, pp.58-66, 2016.

[20] A. Kumar, M. F. Hassan, and P. Raveendran, "Learning based restoration of Gaussian blurred images using weighted geometric moments and cascaded digital filters", Applied Soft Computing, Vol.63, pp.124-138, 2018.

[21] S. Suresh, D. Das, S. Lal, and D. Gupta, "Image quality restoration framework for contrast enhancement of satellite remote sensing images", Remote Sensing Applications: Society and Environment, Vol.10, pp.104-119, 2018.

[22] X. Liu, "Augmented Lagrangian method for total generalized variation based Poissonian image restoration", Computers \& Mathematics with Applications, Vol.71, pp.1694-1705, 2016.

[23] L. Tang, Z. Fang, C. Xiang, and S. Chen, "Image selective restoration using multi-scale variational decomposition", Journal of Visual
Communication and Image Representation, Vol.40, pp.638-655, 2016.

[24] Z. Mbarki, H. Seddik, and E. B. Braiek, "A rapid hybrid algorithm for image restoration combining parametric Wiener filtering and wave atom transform", Journal of Visual Communication and Image Representation, Vol.40, pp.694-707, 2016.

[25] J. Pang, G. Cheung, A. Ortega, and O.C. Au, "Optimal graph Laplacian regularization for natural image denoising", in: Acoustics, Speech and Signal Processing, pp. 2294-2298, 2015.

[26] W. Hu, G. Cheung, A. Ortega, and O.C Au, "Multiresolution graph Fourier transform for compression of piecewise smooth images", IEEE Transactions on Image Processing, Vol. 24, pp. 419-433, 2015.

[27] C. Hu, J. Sepulcre, K.A. Johnson, G.E. Fakhri, Y.M. Lu, and Q. Li, "Matched signal detection on graphs: Theory and application to brain imaging data classification", NeuroImage, Vol.125, pp.587-600, 2016.

[28] W. Q. Zhang, Y. M. Song, and J. Q. Feng, "A New Image Denoising Method Based on Wave Atoms and Cycle Spinning", Journal of Software, Vol.9, pp. 216-221, 2014.

[29] L. Demanet and L. Ying, "Wave atoms and sparsity of oscillatory patterns", Applied and Computational Harmonic Analysis, Vol. 23, pp.368-387, 2007.

[30] K. Dabov, A. Foi, V. Katkovnik, and K. Egiazarian, "Image denoising by sparse 3-D transform-domain collaborative filtering", IEEE Transactions on image processing, Vol.16, No.8, pp.2080-2095, 2007.

[31] A. Buades, B. Coll, and J. M. Morel, "A nonlocal algorithm for image denoising", IEEE Computer Society Conference on Computer Vision and Pattern Recognition, Vol.2, pp.60-65, 2005.

[32] C. Tomasi and R. Manduchi, "Bilateral filtering for gray and color images", In: Proc. of the $6^{\text {th }}$ International Conference on Computer Vision, pp.839-846, 1998. 